\title{
Physicists urge greater efforts on long-range acclerator research
}

HAVE US physicists become so engrossed in the short and medium-term challenges of the design of particle accelerators that they have neglected to give adequate attention to more long-term research needs?

The question is raised empirically by current delays in the production schedules of new accelerators at Fermilab and Brookhaven National Laboratories, at both of which the development of superconducting magnets is presenting more difficulties than originally anticipated.

And it is answered in the affirmative by the Department of Energy's High Energy Physics Advisory Panel (HEPAP) which is about to publish a 'call to arms' to the physics community warning that longrange research is ignored at its peril.

Currently such research consumes less than $1.5 \%$ of the total operating budget of the high energy physics programme. The panel is recommending that this be increased to about $4 \%$ - roughly $\$ 10$ million at current levels - although aware that general budget stringencies make it unlikely that this figure will be realised in the near future.

The report has been prepared by a subpanel of HEPAP. It points out that impressive developments in accelerator technology - frequently based on ideas originating in Europe or the USSR but with their application refined in the US - have produced significant savings in the cost per unit of beam energy. But it also emphasises that the energy demands of physicists have been increasing even faster, roughly by an order of magnitude every seven years.

The net result is that the overall costs of new accelerators continues to rise. Any particular technological advance, such as beam focusing techniques or superconducting magnets, only have a limited effect on reducing costs. "Redoubled efforts" it says are therefore an "essential investment" if further technical breakthroughs are to be found which make the costs of new machines politically acceptable.

The total sums devoted to accelerator $\mathrm{R} \& \mathrm{D}$ are not large. But the money tends to be concentrated on specific projects, with even theoretical work dominated by shortterm goals. $60 \%$ of funds, for example, now go on research on the super-conducting magnets at Fermilab and Brookhaven.

Nor is there necessarily a manpower problem. Of about 1100 high energy physicists in the US, 200 are working fulltime on accelerator research. The report says that the supply of accelerator scientists, although marginal, is not critical.
"We are saying that the mechanisms for accelerator R \& D funding are adequate. What is not adequate is the perception of need in the physics community," Dr Maury Tigner of Cornell University, chairman of the subpanel which produced the report, told HEPAP last week.

"This report is a call-to-arms to the field in general, pointing out that all of us have a problem. Sitting where we are today we can see the beginning of the end of the line of the current generation of accelerators; we want to encourage the community to start thinking about what happens next."

The report emphasises that international comparisons show western Europe and the USSR put more theoretical and experimental effort into long-range accelerator research. "The number of physicists available for this work is larger than in the US, and they are well supported,'” it says.

Among the areas that it suggests might benefit from increased funding are research on very high field superconducting magnets, on radio-frequency superconducting cavities, and on fundamental characteristics such as performance limiting phenomena.

It also recommends a substantial increase in funds for studies of new accelerator concepts. Among, these it lists laser-driven accelerators, and the possible use of linear accelerators with very high beam currents, currently being investigated for their potential use as particle beam weapons, but which have so far received little attention as tools for experimental physicists.

In the near future, however, the chances of increased funding for accelerator R \& D look bleak. The operation of the three national accelerators is already being held back to meet construction commitments on Fermilab's energy saver/doubler and Brookhaven's Isabelle. And the Congress has high energy physics in view as one area to absorb a 'pause' in funding with no shortterm impact on the national economy.

Furthermore the Office of Management and Budget has always been reluctant to consider long-range research proposals, looking on them as, in the words of one energy department official, "the way in which we get pregnant with expensive accelerators". But even some politicians are becoming piqued at the US's loss of leadership to Europe. And this combined with the arguments that accelerator technology can have valuable spin-offs, may provide a counter-balance to budgeteer's fears.

David Dickson

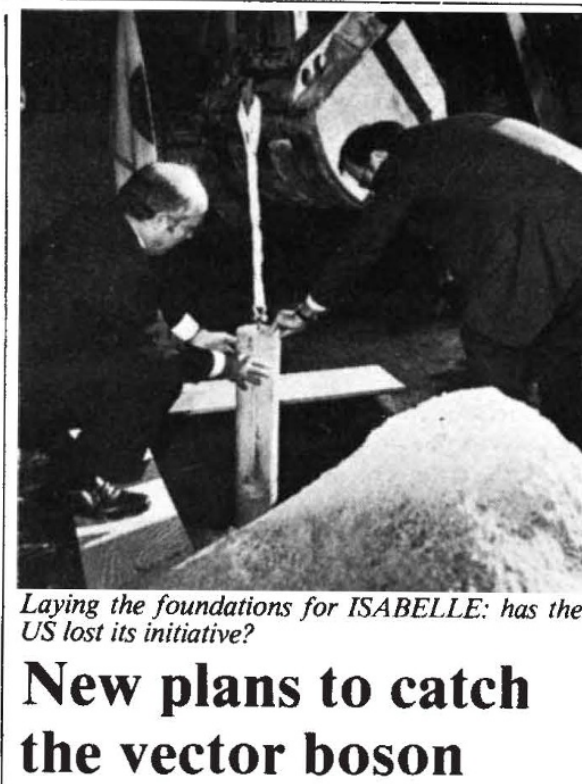

EUROPEAN and American physicists continue to play cat and mouse with their plans for new particle accelerators. First it was over who will get first crack at the $\mathrm{Z}^{0}$ particle, the neutral intermediate vector boson predicted by the Weinberg-Salam unified field theory.

Investigating the characteristics of the $\mathrm{Z}^{0}$ - if it exists - is a prime goal of the Large Electron Position ring (LEP), proposed for construction at the European Centre for Nuclear Research (CERN) with earliest possible completion planned for 1986 .

Rising to the challenge, physicists at the Stanford Linear Accelerator Center worked out a way they could adapt their machine to produce single collisions beteen bunches of electrons and positrons at the $90 \mathrm{GeV}$ range where the $Z^{0}$ is expected to be found, thus beating the Europeans to the mark.

CERN, under strong prompting from its director-general elect Dr Herwig Shopper, responded by pushing forward plans for LEP so that it could become partly operational by 1986 , thereby closing and possibly eliminating the gap with the US.

Now US physicists are suggesting another tack, proposing that if the $Z^{0}$ race is conceded to Europe, it might make more sense to try competing in a different area, namely by constructing a machine to examine electron-proton interactions.

In Europe, where theoretical physicists have been supporting the study of electronproton interactions for some time, such experiments will be carried out on Germany's proposed HERA accelerator in Hamburg. The construction schedule for HERA, however, which has still to be approved by the German government, would start with electron-electron interactions, not bringing the proton ring into operation until 1988 or 1989.

Some US physicists are arguing that, if an electron storage ring can be attached to one of the existing proton accelerators, they could get there considerably sooner, perhaps even by 1983 . 